\title{
New technologies take root in the search for antibiotics from soil
}

Alexander Fleming discovered penicillin in 1928 as a result of a coincidental observation, but modern medicine also owes thanks to the microbiologist Selman Waksman, who subsequently helped develop a platform for studying the antimicrobial activity of various microbes by detecting zones of growth inhibition of susceptible microorganisms on Petri dishes. Waksman and his team used the approach to isolate some 20 new antibiotics from soil microbes, including streptomycin, the first to work against tuberculosis. This method was successfully applied by the pharmaceutical industry for several decades, but when the discovery of new antibiotics dwindled, nobody worried. Science had conquered nature, or so it seemed.

Nothing is farther from the truth, as we know now. A WHO report on antimicrobial resistance published in 2014 suggested that two key antibiotics, fluoroquinolones and carbapenem antibiotics, no longer work in more than half of people taking these drugs in some countries. Unfortunately, efforts to develop high-tech platforms for the discovery of new antibiotic leads have failed. But new technologies-some of which ditch the Petri dish altogether-promise to enable the mining of previously unknown resources and might trigger a new wave of antibiotic discoveries.

Soil microbes heavily compete for territory and resources, using a variety of antimicrobial components. Genetic studies have shown that only $1 \%$ of the microbial soil population can be grown in the lab using conventional culturing techniques. That's because many microbes can't stand oxygen and depend on interactions with plants and other microbes for their survival. Both DNA-based techniques and innovative culturing methods now give scientists the opportunity to screen many more microbes than Waksman and his team could. "We can now go back to see what they've left behind," says Sean Brady, associate professor at the Laboratory of Genetically Encoded Small Molecules at Rockefeller University in New York, whose research focuses on the discovery, biosynthesis and characterization of new, genetically encoded small molecules from microbial sources.

A paper published in early January could herald this Renaissance of antibiotic discovery (Nature 517, 455-459, 2015). An international team of researchers presented a compound called teixobactin, which was shown to be effective against a wide range of Gram-positive bacteria, including methicillin-resistant Staphylococcus aureus. It was produced by a previously unculturable bacteria now named Eleftheria

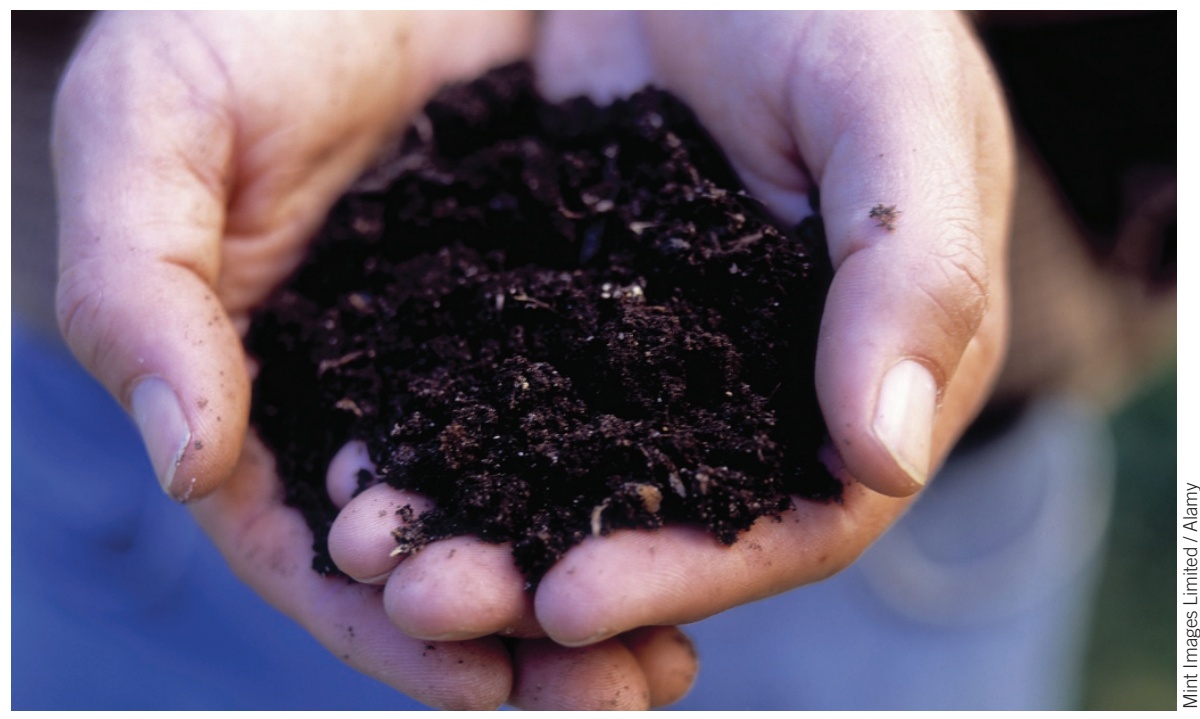

Grounded science: Microbes in the soil may hold the secret to new therapies.

terrae. It was the first discovery of a new major type of antibiotic in 25 years, but the technology used for the research might be even more notable than the discovery itself.

The discovery team, led by Kim Lewis, director of the Antimicrobial Discovery Center at Northeastern University in Boston, developed a device they called iChip, which they literally put into the mud. It was based on a simple idea: if you can't bring the environment to the lab, bring the lab to the environment. The device consists of a platform with holes, in which each hole captures one microbe from the diluted mud, with semi-permeable membranes maintaining contact with the environment in order to keep the captured microbes alive. "Once these single captured cells multiply into colonies, they can be grown in a Petri dish," says Kim Lewis, and thus made ready for use in screening for antimicrobial activity.

\section{Get their genes out}

There are other approaches on the horizon as well. Brady and his colleagues, for instance, recently published a method based on a very different principle: if you can't culture the microbes, get their genes out (eLife, 10.7554/eLife.05048, 2015). They analyze all the microbial genetic material from a soil sample and search for genes encoding potential new antibiotics. This has one clear advantage over culturing: even when microbes can be cultured, they will not produce all the molecules their genes enable them to produce, because many genes are switched off. Presumably, they need environmental triggers such as signals from other microbes or plants to switch them on and awaken the production of antibiotics. Brady and colleagues clone the genes encoding these potentially useful molecules into model organisms such as $E$. coli. If the organism does not begin producing the compound spontaneously, the team replaces the gene promoter with one that does start the process.

Whereas the iChip has proven to be a great tool for the discovery of novel compounds, Brady's strategy particularly enables the search for variants of existing antibiotics. They use primers for known gene clusters to screen for these and comparable sequences. They have identified a number of new molecules and hot spots of additional interesting genes that they will test in model organisms. "The answers to antibiotic resistance should be out there in nature," says Brady. "Our method speeds up the process of finding these."

In the meantime, other groups are taking different approaches. Some attempt to trigger cultured bacteria to switch on a greater proportion of their genes, combining the advantages of Lewis's and Brady's approaches. Yet others search for methods to trace the molecules that are produced in small amounts in fermentation flasks, which are usually overshadowed by the more-abundant compounds.

Taken together, these technologies create ample opportunities for antibiotic discovery. The dropout rate will undoubtedly be high, mainly owing to human toxicity issues, but at least there will presumably be new compounds to be tested in follow-up studies. Lewis, in any case, predicts more discoveries from his group: "We will continue our screening program, and hope to up-scale it in the upcoming months."

Jop de Vrieze

Corrected after print 7 May 2015. 


\section{Correction}

An earlier version of the article "New

technologies take root in the search for antibiotics from soil" (Nat. Med. 21, 201,

2015) inaccurately stated that researchers

have not identified any new molecules using

iChip technology. In fact, this technology

has found new molecules and genes of

interest. The error has been corrected in the

HTML and PDF versions of the article. 